\title{
Catecholamine Toxicity in Cerebral Cortex in Dissociated Cell Culture
}

\author{
Paul A. Rosenberg \\ Department of Neurology, Children's Hospital and Harvard Medical School, Boston, Massachusetts 02115
}

\begin{abstract}
Identification of endogenous toxins and characterization of the mechanisms by which toxins produce cell injury and death may help understand both normal modeling of cell populations and connections in the CNS as well as abnormal cell loss. The toxicity of catecholamines intrinsic to the CNS was investigated using the model system of rat cerebral cortex in dissociated cell culture. All catecholamines tested, including norepinephrine (NE), dopamine, and epinephrine, were toxic to neurons as well as glia at a concentration of $25 \mu \mathrm{m}$ when added to cultures $24 \mathrm{hr}$ after plating. Toxicity was evident after $48 \mathrm{hr}$ exposure to $\mathrm{NE}$, as monitored by loss of cells from the cultures. Toxicity did not seem to be mediated by adrenergic receptors because, although the beta-adrenergic agonist isoproterenol (but not the alphaadrenergic agonist phenylephrine) was similar in its toxic effect to NE, the beta-adrenergic antagonist atenolol did not block the toxic effect of NE. Toxicity could be mimicked by hydrogen peroxide, a product of the oxidative degradation of catecholamines. Toxicity of NE was blocked by catalase. The neurotoxin 6-hydroxydopamine (6-OHDA), supposedly selective for catecholaminergic neurons, was found to be toxic over the same concentration range as NE. These results suggest that endogenous catecholamines may play a role in normal and abnormal cell death, and suggest that caution be used in relying on the specificity of 6-OHDA and other supposedly selective neurotoxins.
\end{abstract}

The potential importance of neurotoxins derived from either endogenous or exogenous sources in the pathogenesis of neurological disease has been underscored by recent observations (Olney, 1978; Langston et al., 1983; Choi, 1985; Ferrante et al., 1985; Javitch et al., 1985; Rothman, 1985; Beal et al., 1986; D'Amato et al., 1986). Interest in the toxicity of catecholamines has been largely restricted to the selective destruction of catecholaminergic neurons by 6-hydroxydopamine (6-OHDA) (Malmfors and Thoenen, 1971). This compound accumulates within catecholamine nerve terminals after injection into experimental animals and subsequently causes destruction of these nerve terminals and loss of catecholaminergic neurons. The extent of the depletion of catecholamines depends upon the animal, age, and route of administration. The mechanism of

\footnotetext{
Received July 28, 1987; revised Oct. 22, 1987; accepted Nov. 23, 1987.

This work was supported by a Robert Morison Fellowship from the Grass Foundation, a Clinical Investigator Development Award, NS 00993, and Children's Hospital Mental Retardation Core HD 06276. The author wishes to thank Dr. Marc Dichter for use of laboratory facilities and for helpful discussions, and Sara Vasquez for excellent technical assistance.

Correspondence should be addressed to Dr. Paul A. Rosenberg, Enders 4, Department of Neurology, Children's Hospital, 300 Longwood Avenue, Boston, MA 02115.
}

Copyright (C) 1988 Society for Neuroscience $0270-6474 / 88 / 082887-08 \$ 02.00 / 0$ this toxicity is assumed to be a consequence of autoxidation and the generation of toxic quinones and hydrogen peroxide. Nonspecific toxicity at sites of injection of 6-OHDA is well known (Ungerstedt, 1971).

The possibility that catecholamines may cause damage to noncatecholaminergic neurons, or to glia, has not received much attention, although it has been considered (Graham, 1978, 1984; Marker et al., 1981; Suter and Matter-Jaeger, 1984). On the other hand, there is a large body of literature on the possibility of both receptor-dependent and receptor-independent mechanisms of catecholamine cardiotoxicity (Wheatley et al., 1985). This report describes toxicity of catecholamines, including 6-OHDA, occurring at micromolar concentrations to noncatecholaminergic neurons as well as glia in the model system of rat cerebral cortex in dissociated cell culture.

\section{Materials and Methods}

Neocortical cultures were prepared as reported by Dichter (1978; Snodgrass et al., 1980). Cortices were dissected from CD rat embryos at embryonic day (E) 15. The tissue was then incubated in Earle's salt solution containing $0.03 \%$ trypsin for $2 \mathrm{hr}$ at $37^{\circ} \mathrm{C}$ and then calciumand magnesium-free Earle's salt solution for an additional $20 \mathrm{~min}$ at $37^{\circ} \mathrm{C}$. The tissue was triturated and then filtered through sterile lens paper to remove debris and cell clumps. The suspension was diluted with minimum essential medium (MEM) supplemented to $200 \mathrm{mg} \%$ glucose, penicillin $(20 \mathrm{U} / \mathrm{ml})$, streptomycin $(20 \mu \mathrm{g} / \mathrm{ml})$, and $5 \%$ rat serum heat-inactivated at $56^{\circ} \mathrm{C}$ for $30 \mathrm{~min}$. It was found over many dissections and platings of cultures that a given plating density yielded widely varying numbers of surviving cells per culture. Plating density was 225,000 or 450,000 trypan blue-excluding cells $/ 35 \mathrm{~mm}$ dish $(1.5 \mathrm{ml}$ plating volume), producing cultures with $150-400$ neurons/ 10 fields at $400 \times$. Each $35 \mathrm{~mm}$ dish contained 5 coverslips that had been precoated with poly-L-lysine. Coverslips were precoated by incubating them for $24 \mathrm{hr}$ in a $1 \mathrm{mg} / \mathrm{ml}$ solution of poly-L-lysine (Peninsula) in $0.1 \mathrm{M}$ borate buffer, $\mathrm{pH} 8.4$, followed by 2 washes, $24 \mathrm{hr}$ each, with distilled water. Dishes with coverslips were incubated for $24-48 \mathrm{hr}$ with growth medium plus $5 \%$ fetal calf serum (instead of rat serum) prior to plating cultures.

At $24 \mathrm{hr}$, coverslips were distributed into the wells of 24-well plates (Costar). To each well receiving a coverslip was first added $0.5 \mathrm{ml}$ of the growth media. The 5 coverslips of each dish were distributed among all 5 experimental conditions, including the control condition (or, for time course experiments, the zero-time condition), so that one coverslip from each dish served as the control for the remaining coverslips of the dish. For most experiments, except as noted, conditions were replicated in triplicate. After coverslip cultures were placed in wells with media, drugs or vehicle were added. Catecholamine solutions were prepared from $10 \mathrm{~mm}$ stocks made up with a catecholamine vehicle $\left(10^{-3} \mathrm{M} \mathrm{HCl}\right.$ and $10^{-4} \mathrm{M}$ EDTA, filtered using Millipore $0.45 \mu \mathrm{m}$ HA filters). Tenfold dilutions from this stock were made using this vehicle $(1 \mathrm{~mm}, 0.1 \mathrm{~mm}$, $0.01 \mathrm{~mm}$ ). To each well was added $12.5 \mu 1$ of either the appropriate drug solution or vehicle, for a final 41-fold dilution into growth medium. The final concentrations of the components of the vehicle were $25 \mu \mathrm{M}$ $\mathrm{HCl}$ (without significant cffect on the $\mathrm{pH}$ of the medium, buffered with $26.2 \mathrm{~mm}$ bicarbonate) and $2.5 \mu \mathrm{M}$ EDTA (without significant effect on concentration of divalent cations, present in millimolar quantities). Experiments were also performed using a vehicle of $10^{-3} \mathrm{M} \mathrm{HCl}$, with similar results. Stable adrenergic agonists were made up in distilled water 
for most experiments, but results were the same when the catecholamine vehicle was used. After additions, wells were briefly agitated and were placed in the incubator.

At $72 \mathrm{hr}$, cultures were stained with trypan blue and then fixed, exploiting the observation that trypan blue is fixed by glutaraldehyde (Pixley and Cotman, 1985). Media were removed and replaced with 0.5 $\mathrm{ml}$ of a $1: 1$ dilution of trypan blue $(0.4 \%$ in $0.9 \% \mathrm{NaCl}$; Sigma) with physiological saline ( $\mathrm{NaCl}, 145 \mathrm{~mm} ; \mathrm{KCl}, 3 \mathrm{~mm} ; \mathrm{CaCl}_{2}, 1.8 \mathrm{~mm} ; \mathrm{MgCl}_{2}$, $1 \mathrm{~mm}$; glucose, $8 \mathrm{~mm}$; $\left.\mathrm{NaH}_{2} \mathrm{PO}_{4}, 2.4 \mathrm{~mm} ; \mathrm{Na}_{2} \mathrm{HPO}_{4}, 0.42 \mathrm{~mm}\right)$. Cultures were incubated with trypan blue for $1 \mathrm{~min}$, trypan blue was removed, cultures were washed twice with $0.5 \mathrm{ml}$ physiological saline, and then $2.5 \%$ glutaraldehyde in physiological saline was added. Cultures were fixed for $1 \mathrm{hr}$ and then washed, dehydrated in ethanol, cleared in Histoclear (National Diagnostics), and mounted in Permount. Cells were counted in 10 fields at $400 \times$, which represented $1.4 \%$ of the total area of the coverslip. In some, but not all, experiments, cells were present that were not stained by trypan blue but were small, phase-dark, round, without processes, and vacuolated. Cells that were not blue and that were not as just described were counted as live cells (live at the time of exposure to trypan blue). Cells were counted either as neurons, having neuronal morphology, or flat cells. All experiments were repeated at least 3 times. Student's 2-tailed, unpaired $t$ test was used to evaluate the statistical significance of experimental results. The Bonferroni correction was used to correct for multiple comparisons.

Stock solutions of catecholamines were stored at $4^{\circ} \mathrm{C}$. The stability of norepinephrine (NE) under the conditions of storage was confirmed by direct assay of NE in stock solutions, using a radioenzymatic procedure (Upjohn). NE solutions were stable for months under these conditions. 6-OHDA solutions were made up fresh for experiments, using the catecholamine vehicle. Norepinephrine hydrochloride, dopamine hydrochloride, epinephrine bitartrate, serotonin creatinine sulfate, and 6-hydroxydopamine hydrochloride were the salts used; they were obtained from Sigma.

Cultures were characterized with respect to their cellular composition using antibodies directed against cell-specific antigens, with distribution restricted to particular types of cells, as well as with tetanus toxin, which specifically labels plasma membranes of all neurons in culture (Raff et al., 1979). The following antibodies were used: monoclonal anti-vimentin (Boehringer-Mannheim), a marker for cells of mesenchymal origin (Dahl et al., 1981), as well as some, but not all, astrocytes (Franke et al., 1978); polyclonal anti-glial fibrillary acidic protein (GFAP) (courtesy A. Bignami); and human anti-tetanus immune globulin (Cutter). Tetanus toxin was kindly donated by the Massachusetts Department of Public Health.

In all cases, cell-marking experiments were performed on 4-d-old cultures that had been placed in fresh growth medium in wells at $24 \mathrm{hr}$ exactly analogous to the procedure used for the catecholamine toxicity experiments. In experiments using anti-vimentin and anti-GFAP, 18 coverslip cultures from 3 different culture dates were stained: $15.4 \pm$ $8 \%$ of cells were vimentin-positive, $1.7 \pm 0.8 \%$ of cells were GFAPpositive, and $1.2 \pm 0.7 \%$ of cells were double-labeled with the 2 antibodies. Vimentin-positive cells were of 3 types: small flat cells, 10-20 $\mu \mathrm{m}$ diameter, which were GFAP-negative; medium-size, 20-30- $\mu \mathrm{m}$ diameter flat cells, some of which were GFAP-positive; and large, very flat cells greater than $30 \mu \mathrm{m}$ in diameter, which were GFAP-negative. Cells that were labeled neither with anti-vimentin nor with anti-GFAP antibodies had a neuronal morphology: 1-3 thin (less than $1 \mu \mathrm{m}$ across), tapering processes from a pyramidal or bipolar-shaped cell body with a large nucleus. Since it is known that some neurons stain with antivimentin (Bignami et al., 1982), and since, in fact, in these cultures some cells that were stained with anti-vimentin had a neuronal morphology and were GFAP-negative, it is likely that some vimentin-containing cells were neurons. Therefore, the total of vimentin-positive plus GFAP-positive cells gives, if anything, an overestimate of the number

Figure 1. Effect of NE on cerebral cortex in culture. Coverslip cultures of rat cerebral cortex were prepared as described. At $24 \mathrm{hr}$, coverslips were placed in wells containing $0.5 \mathrm{ml}$ growth medium. Catecholamine vehicle or drug solution, $12.5 \mu \mathrm{l}$, was added to each well. The plate was briefly agitated and then incubated at $37^{\circ} \mathrm{C}$ for $72 \mathrm{hr}$. The medium was removed and cultures incubated in a $1: 1$ dilution of $0.4 \%$ trypan blue with physiological saline for $1 \mathrm{~min}$. Cultures were washed and fixed with $2.5 \%$ glutaraldehyde for $1 \mathrm{hr}$, then washed and mounted. Cultures
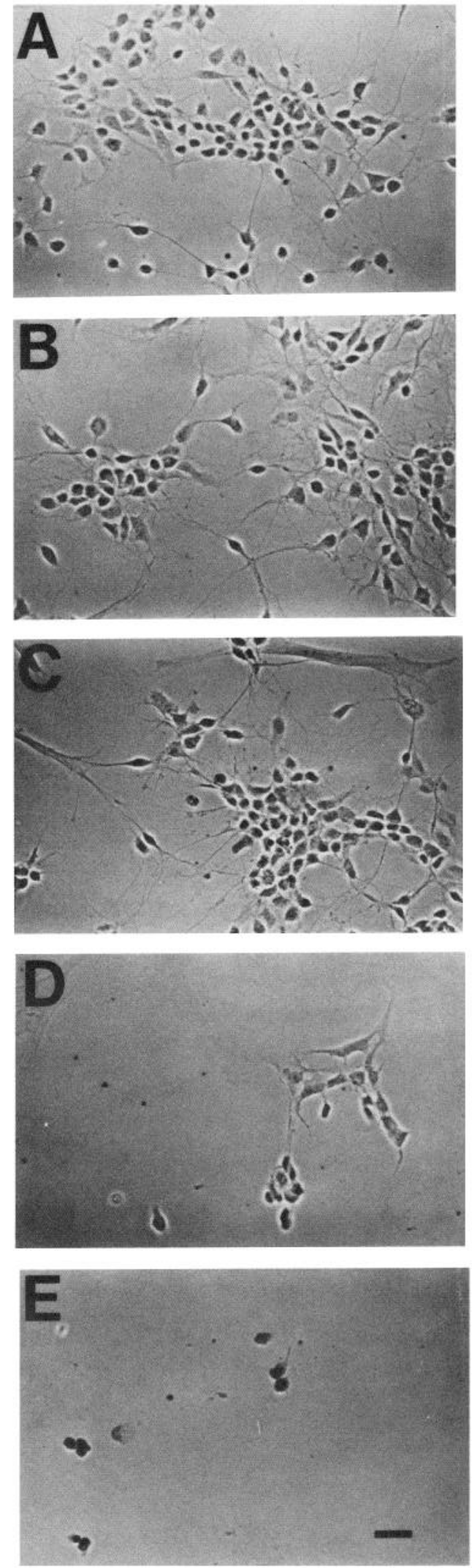

of cerebral cortex were exposed to NE at final concentrations of 0.25 $\mu \mathrm{M}(B), 2.5 \mu \mathrm{M}(C), 25 \mu \mathrm{M}(D), 250 \mu \mathrm{M}(E)$, or to vehicle only $(A)$. Phasecontrast optics were used to photograph the trypan blue-stained, fixed, and mounted cultures that were counted to produce the data displayed in Figure 2. Dark cells in $250 \mu \mathrm{M}$ NE $(E)$ were trypan blue-stained. Scale bar, $40 \mu \mathrm{m}$. 


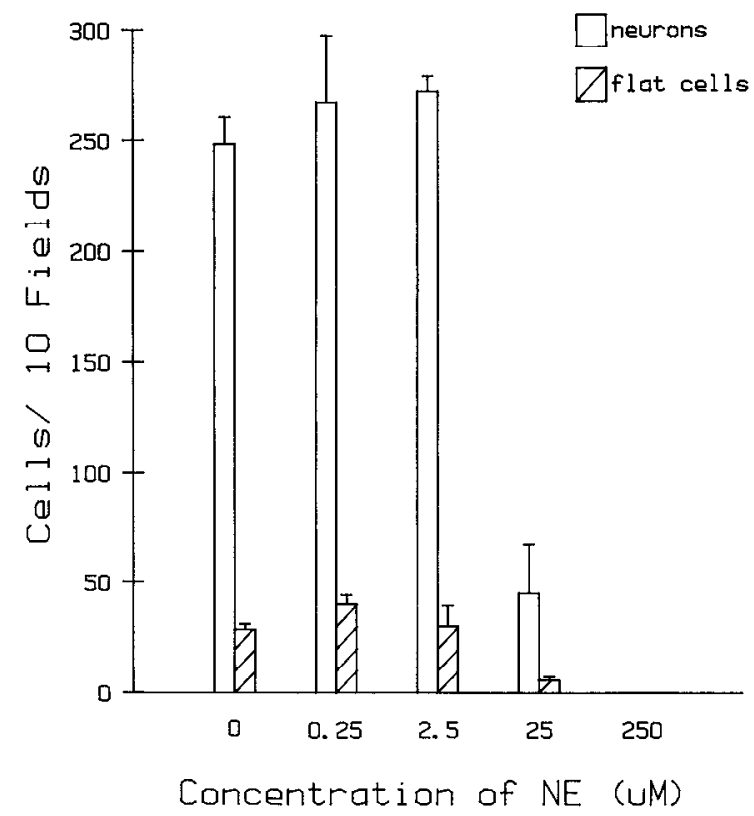

Figure 2. NE toxicity: dose-response. Ten nonoverlapping fields/coverslip were counted, scoring neurons and flat cells that were not stained by trypan blue, and that therefore were presumed to have been alive at the time of exposure to this stain, which does not enter live cells. In catecholamine vehicle alone, blue cells represented $1.7 \%$ of total cells counted. In $250 \mu \mathrm{M}$, only blue cells were present, with an average of 56 counted per coverslip. In other experiments, no differences were noted in the number of blue cells present, counting trypan blue-treated cultures prior to fixation or after fixation and mounting. NE had no effect at 2.5 $\mu \mathrm{M}$, but at $25 \mu \mathrm{M}$ the number of neurons surviving at $72 \mathrm{hr}$ fell to $18 \%$ of control $(p<0.001)$. The number of flat cells surviving at this concentration fell to $21 \%$ of control cultures $(p<0.001)$. There were no surviving cells in $250 \mu \mathrm{M} \mathrm{NE}$. In other experiments it was shown that vehicle alone had no effect on the survival of cells in the cultures. Error bars indicate SDs in this and subsequent tigures.

of non-neuronal cells in culture. However, since many vimentin-positive cells are clearly non-neuronal morphologically and are GFAP-negative, GFAP alone is not sufficient to characterize the number of nonneuronal cells in the culture. Staining with tetanus toxin at this stage yields distinct staining of the plasma membranes of neurons in a punctate pattern characteristic of tetanus toxin, and clearly distinguishes neurons from non-neuronal cells. In 3 separate experiments using 4-dold cultures, tetanus toxin labeled $57.8 \pm 5.1 \%$ of cells $(n=9$ coverslip cultures counted).

\section{Results}

$\mathrm{NE}$ at a concentration of $25 \mu \mathrm{M}$ was associated with a large loss of cells from the culture after $72 \mathrm{hr}$, evident by visual inspection of the cultures (Fig. 1). No trypan blue-stained cells are present in Figure 1, $A-D$, typical for this culture system, in that dead cells seem to disintegrate rapidly and disappear from the cultures, rather than persist as trypan blue-including cells. For Figure 2, the cultures shown in Figure 1 were counted and the results plotted. In this representative experiment, only $18 \%$ of the number of cells with neuronal morphology present in control cultures survived. No effect of NE was seen at $2.5 \mu \mathrm{M}$, and no cells were left on the coverslip at $250 \mu \mathrm{M}$, at which concentration, after $24 \mathrm{hr}$, the medium (which contained phenol red) acquired a brownish color, presumably from the presence of oxidation products of NE. In addition to the loss of neurons at $25 \mu \mathrm{M} \mathrm{NE}$, this concentration of NE also produced a comparable loss of flat, non-neuronal cells, with $21 \%$ remaining at $25 \mu \mathrm{M}$. Surviving cells were always rare in $250 \mu \mathrm{M}$ NE. The fraction of cell loss

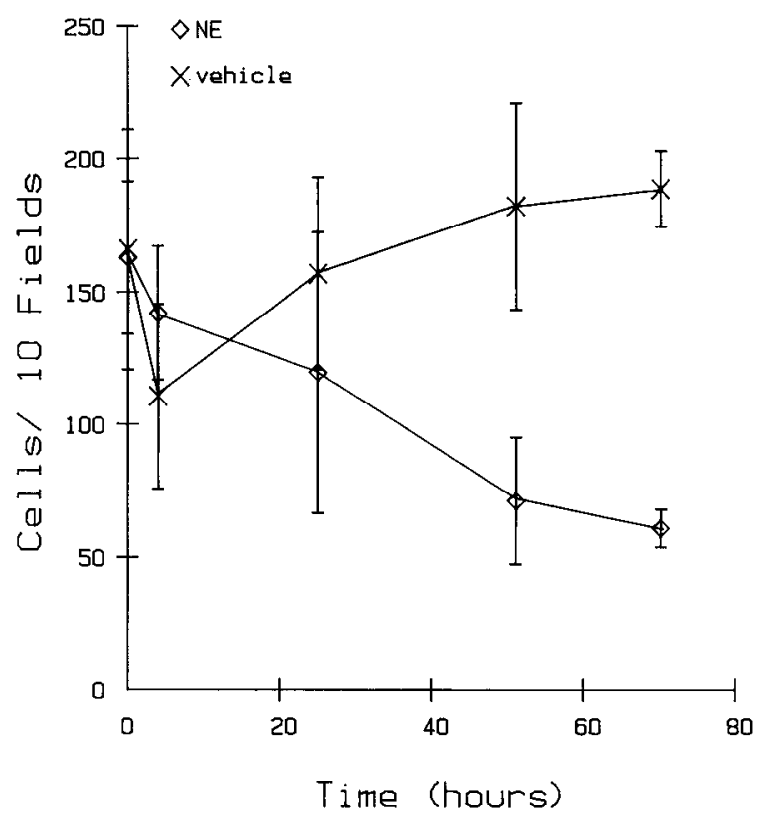

Figure 3. Time course of NE toxicity. Cultures were placed in growth medium with $25 \mu_{\mathrm{M}} \mathrm{NE}$ (diamonds) in vehicle, or with vehicle only ( $\times$ s). At $0,4,25,51$, and $70 \mathrm{hr}, 3$ coverslips were removed, stained with trypan blue, fixed, and counted. There was a steady decline in the number of surviving neurons, with the greatest loss occurring in the first $50 \mathrm{hr}$. By $70 \mathrm{hr}$, only $37 \%$ of neurons present at the beginning of the experiment had survived. Cultures with vehicle only were sampled at the same time as the cultures with NE. Note that the number of neurons in thesc cultures was stable during the experiment, with no significant change in number appreciable.

at $25 \mu \mathrm{M}$ was variable from culture to culture, and, as will be demonstrated subsequently, was dependent on culture density.

Experiments were performed to investigate the time course of the toxic effect of NE (Fig. 3). In these experiments, cultures were exposed to growth medium with $25 \mu \mathrm{M}$ NE or with vehicle only for varying intervals of time, and were then removed for observation. At approximately $0,4,24,48$, and $72 \mathrm{hr}$, a group of cultures was removed, incubated with trypan blue, fixed, and then counted. Cultures in the presence of NE showed a steady decline in the number of neurons present for at least over 48 hr (Fig. 3, diamonds). Control cultures, exposed to growth medium plus vehicle, showed no loss in the number of neurons or non-neuronal cells present (Fig. $3, \times$ s).

In another series of experiments, the effect of varying the exposure time to NE on NE's toxic effect assayed at $72 \mathrm{hr}$ was investigated (Fig. 4). Cultures were placed in medium that contained either 25 or $250 \mu \mathrm{M}$ NE or vehicle only. After $0,1,4$, 24 , or $72 \mathrm{hr}$, this medium was removed and replaced with fresh medium. For these experiments, the usual growth medium could not be used because it was found that, using growth medium, a second medium change within $72 \mathrm{hr}$ was itself toxic to the cultures. This effect was found to be due to the rat serum. Medium change within $72 \mathrm{hr}$ with growth medium without rat serum did not have a toxic effect on the cultures. This experiment was therefore conducted using growth medium without rat serum.

In $25 \mu \mathrm{M}$ NE (Fig. 4, diamonds), no significant differences were observed in the number of neurons remaining at $72 \mathrm{hr}$ with exposures lasting $0,1,4$, or $24 \mathrm{hr}$. All neurons were killed by a $72 \mathrm{hr}$ exposure. In $250 \mu \mathrm{M} \mathrm{NE}$, an effect was apparent following a $1 \mathrm{hr}$ exposure, with $28 \%$ of the neurons remaining 


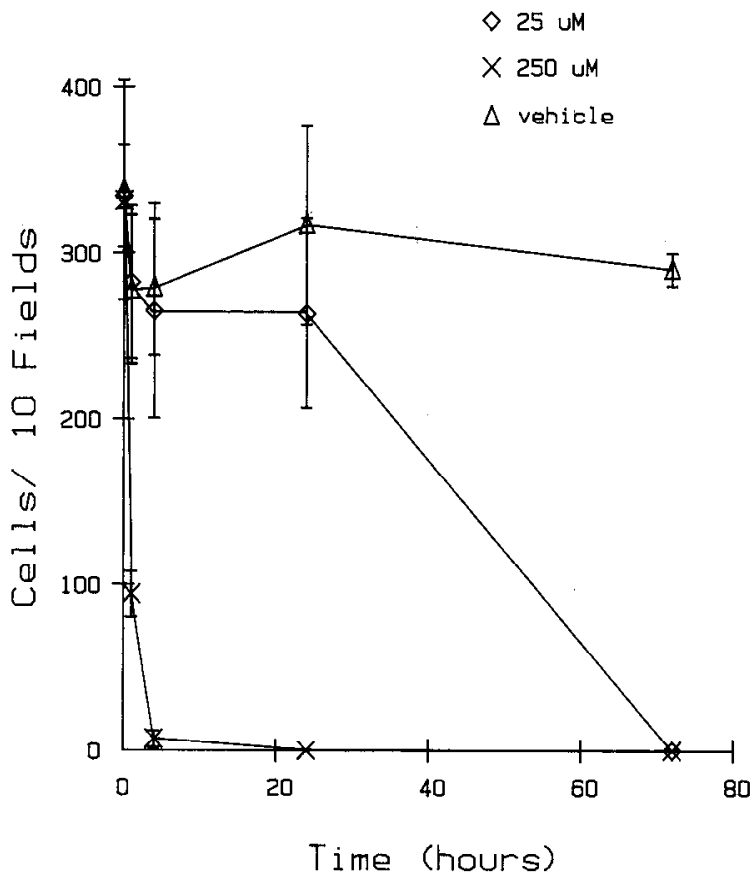

Figure 4. Effect of varying length of exposure to NE on survival. In this experiment, cultures were exposed to $25 \mu \mathrm{M} \mathrm{NE}$ (diamonds), 250 $\mu \mathrm{M} N E(\times \mathrm{s})$, or vehicle only (triangles) for $0,1,4,24$, and $72 \mathrm{hr}$, and the effect on survival of neurons at $72 \mathrm{hr}$ observed. Growth medium without serum was used for reasons described in the text. With exposures up to $24 \mathrm{hr}$, no differences were observed between cultures exposed to $25 \mu \mathrm{M}$ NE and those exposed to catecholamine vehicle only. A $72 \mathrm{hr}$ exposure was required to produce the maximum toxic effect of NE at $25 \mu \mathrm{M}$. In contrast, at $250 \mu \mathrm{M} \mathrm{NE}$, a large reduction in surviving neurons was observed following a $1 \mathrm{hr}$ exposure (28.4\%). The population of neurons in the culture was stable over the course of the experiment in control cultures.

at $72 \mathrm{hr}$. With a $4 \mathrm{hr}$ exposure, only $2 \%$ of the neurons remained at $72 \mathrm{hr}$. In contrast, control cultures showed a stable population of neurons over the same period (Fig. 4, triangles).

To test the effect of cell density on the toxicity of catecholamines, experiments were performed in which a dose-response paradigm of exactly the type described for Figures 1 and 2 was performed on cultures plated at 2 culture densities, 450,000, and 225,000 cells/dish (Fig. 5). At the lower plating density, the effect of $25 \mu \mathrm{M}$ NE was devastating, with no neurons and few flat cells remaining. At the higher density, there was no significant effect of NE at $25 \mu \mathrm{M}$. At $250 \mu \mathrm{M}$, no cells survived.

In order to understand the mechanism of NE toxicity, it was necessary to determine whether this phenomenon was mediated by adrenergic receptors or not. To test whether NE toxicity was mediated by adrenergic receptors, experiments were performed in which cultures were exposed either to $0,0.25,2.5,25$, and $250 \mu \mathrm{M} \mathrm{NE}$, to the beta-adrenergic agonist isoproterenol, or the alpha-adrenergic agonist phenylephrine, all in growth medium (Fig. 6). At $25 \mu \mathrm{M}$, both NE and isoproterenol significantly reduced the number of neurons remaining in the cultures at 72 $\mathrm{hr}$, as compared to their controls. The number of cells remaining in $25 \mu \mathrm{M}$ phenylephrine was not significantly different from its control. At $250 \mu \mathrm{M}$, no neurons remained in cultures exposed to NE or isoproterenol. At this concentration of phenylephrine, there was a reduction in the number of neurons remaining, to $47.5 \%$ of control, which, however, still did not attain statistical significance. This result suggests that if the toxic effect of NE

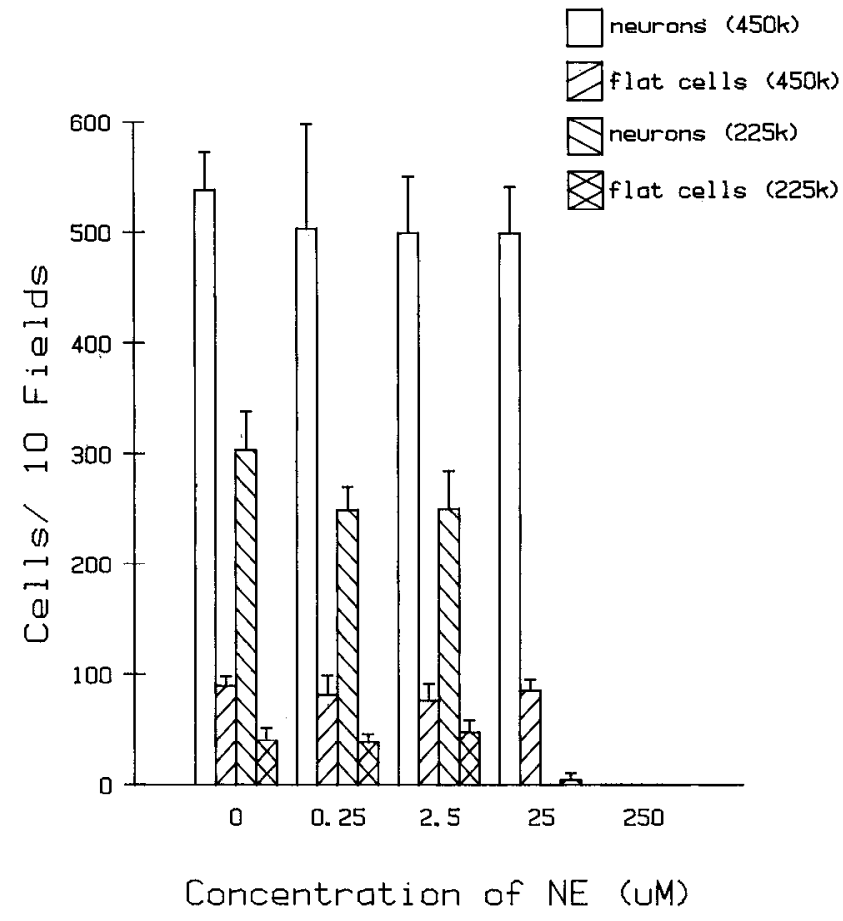

Figure 5. Effect of cell density on the toxicity of NE. Cortical cultures were plated at 2 densities, 450,000 , and 225,000 cells $/ 35 \mathrm{~mm}$ culture dish. Plating volume was $1.5 \mathrm{ml}$. Cultures at the 2 densities were set up in a concentration series of NE, as described for Figure 1. This experiment was performed in quadruplicate. At neither density was an effect of $2.5 \mu \mathrm{M}$ NE observed. At the lower density, no neurons survived after $72 \mathrm{hr}$ in $25 \mu \mathrm{M}$ NE. At 450,000 cells per dish, there was no significant loss of cells at this concentration of NE. No cells survived in $250 \mu \mathrm{M}$ NE at either plating density.

were mediated by an adrenergic receptor, the effect would be primarily mediated by a beta-adrenergic receptor, but it does not exclude a contribution by alpha-adrenergic receptors.

If NE toxicity were mediated predominantly by beta-adrenergic receptors, then a beta-adrenergic antagonist should block the effect. Cultures were incubated for $72 \mathrm{hr}$ with either $25 \mu \mathrm{M} \mathrm{NE}$, $250 \mu \mathrm{M}$ atenolol, NE plus atenolol, or vehicle only (Fig. 7). Atenolol had no significant effect on its own, and did not block the toxic effect of NE. The finding that atenolol failed to block the toxic effect of NE is inconsistent with the mediation of this toxic effect by a beta-adrenergic receptor. If NE toxicity is not mediated by adrencrgic receptors, another possibility might bc that it is mediated through toxic by-products of the oxidation of NE. Oxidative degradation of catecholamines may proceed by several routes, including auto-oxidation, MAO-catalyzed deamination, and catechol-O-methyl transferase-catalyzed $O$-methylation. Both auto-oxidation and MAO degradation yield hydrogen peroxide stoichiometrically (Senoh and Witkop, 1959; Senoh et al., 1959; Saner and Thoenen, 1970; Heikkila and Cohen, 1973; Liang et al., 1976; Graham, 1978, 1984; Marker et al., 1981). In addition, auto-oxidation of catecholamines yields potentially toxic quinones that are themselves unstable, namely, adrenochrome in the case of epinephrine (EPI), and noradrenochrome and aminochrome in the case of $\mathrm{NE}$ and dopamine (DA), respectively (Heacock, 1959; Graham, 1978). Therefore, a likely mechanism for NE toxicity is auto-oxidation and the production of these reactive intermediates. If this were the case, then one would expect that NE toxicity would be mimicked by hydrogen peroxide or the quinoid by-products at approximately 


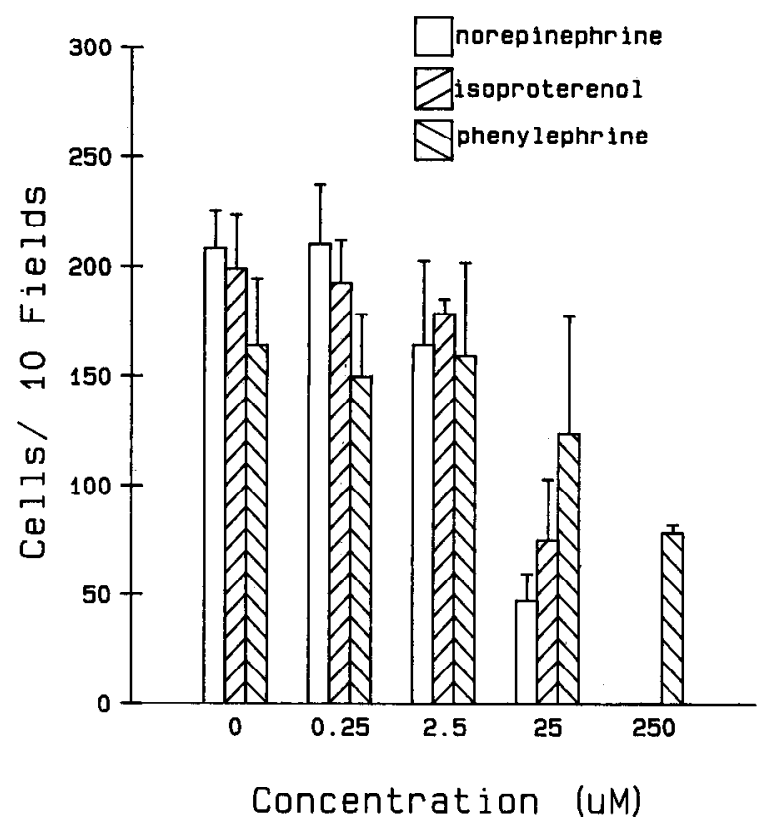

Figure 6. Effect of adrenergic agonists on neuronal survival. In this experiment, the toxic effects of $\mathrm{NE}$, isoproterenol (a beta-adrenergic agonist), and phenylephrine (an alpha-adrenergic agonist) were compared. For each agonist, cultures were exposed to catecholamine vehicle or increasing concentrations of drug, following the same paradigm as in Figure 1. At $72 \mathrm{hr}$, cultures were stained, fixed, and counted. The data for neurons are presented. All cultures were exposed to the same vehicle. At $25 \mu \mathrm{M} \mathrm{NE}, 23 \%$ of the neurons survived, compared to control cultures $(p<0.004)$. At $25 \mu \mathrm{M}$ isoproterenol, $38 \%$ neurons survived, compared to control cultures $(p<0.04$ ). At $25 \mu \mathrm{M}$ phenylephrine, $76 \%$ neurons survived ( $p>0.05$; not significant). At $250 \mu \mathrm{M}$, no neurons remained in cultures exposed to NE or isoproterenol. In cultures exposed to phenylephrine, $47.5 \%$ of neurons survived $(p>0.05)$. The greater potency of isoproterenol than phenylephrine suggests that if NE toxicity were mediated by adrenergic receptors, toxicity would be primarily dependent on the interaction of NE with beta-adrenergic receptors.

the same concentration range at which NE is itself toxic, since they are produced stoichiometrically from NF during oxidation. The toxicity of adrenochrome and hydrogen peroxide, along with EPI, was investigated by incubation with concentrations of EPI or hydrogen peroxide from 0 to $250 \mu \mathrm{M}$ and adrenochrome from 0 to $25 \mu \mathrm{M}$ for $72 \mathrm{hr}$ (Fig. 8). Both hydrogen peroxide and adrenochrome mimicked the effect of EPI at 25 $\mu \mathrm{M}$, suggesting that either or both together might be contributing to the toxicity of EPI.

The possibility that hydrogen peroxide is the toxic intermediate in catecholamine toxicity was tested by experiments in which catalase was used to block this toxicity (Fig. 9). Cultures were exposed either to vehicle, $25 \mu \mathrm{M} \mathrm{NE}$, or $25 \mu \mathrm{M}$ NE plus 10 micrograms $/ \mathrm{ml}$ catalase. Catalase completely blocked the toxicity of NE on both neurons, as well as on flat cells, consistent with the hypothesis that NE toxicity is mediated by the production of hydrogen peroxide from the oxidative degradation of NE. Catalase $(25 \mu \mathrm{cg} / \mathrm{ml})$ was also able to block the toxicity of adrenochrome $(25 \mu \mathrm{M})$. Thus, in a representative experiment, $626 \pm 89$ neurons survived in control cultures; $78 \pm 28$ survived in the presence of adrenochrome; $538 \pm 104$ survived in the presence of catalase; and $445 \pm 84$ survived in the presence of adrenochrome plus catalase. Superoxide dimutase and $d$ - $\alpha$-tocopherol were both tested for their ability to block NE toxicity, but were found to give inconsistent results. Ascorbate at $25 \mu \mathrm{M}$ did not affect toxicity.

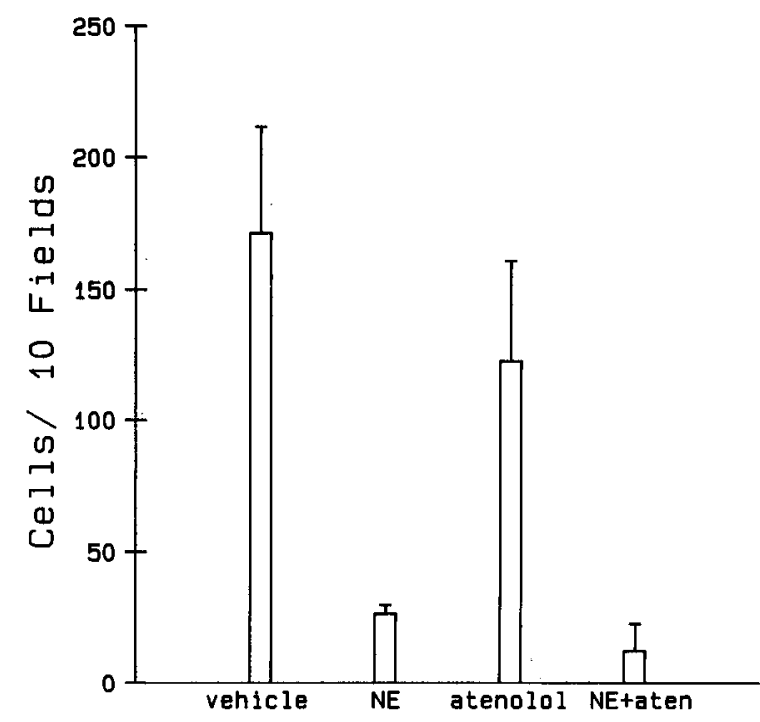

Figure 7. Effect of atenolol on NE toxicity. Cultures were exposed to either vehicle, NE $25 \mu \mathrm{M}$, atenolol $250 \mu \mathrm{M}$, or NE $25 \mu \mathrm{M}$ plus atenolol $250 \mu \mathrm{M}$. All cultures were exposed to the same vehicle. Data for neurons only are shown. Atenolol by itself had no significant effect on the cultures at this concentration, as shown here. NE reduced surviving neurons to $15.3 \%$ of control (vehicle only) $(p<0.03)$. The addition of atenolol had no effect on the toxicity of NE (no significant difference between $N E$ and $N E+$ aten).

Experiments were performed in which the toxicity of NE, DA, and serotonin was compared (Fig. 10). Cultures were exposed to $0,0.25,2.5,25$, and $250 \mu \mathrm{M}$ monoamine in growth medium for $72 \mathrm{hr}$. At $25 \mu \mathrm{M}$, both NE and DA drastically reduced the number of neurons remaining in the cultures, while serotonin had no effect. At $250 \mu \mathrm{M}$ serotonin, there was a reduction in the number of neurons remaining in the cultures to about $30 \%$ of controls. All catecholamines tested had comparable toxicity, a property not shared by serotonin, a more stable and noncatecholaminergic monoamine.

Similar experiments testing the toxicity of 6-OHDA demonstrated that this compound also had a toxic effect on cultures comparable to that seen with the catecholamines. Again, $25 \mu \mathrm{M}$ was the lowest concentration at which toxicity was manifest in terms of cell survival, with loss of nearly all neurons from the cultures ( 4 experiments, no neurons remaining in 2 and less than $2.5 \%$ remaining in 2 ). Flat cells were similarly affected. No toxicity was observed at $2.5 \mu \mathrm{M} 6$-OHDA.

\section{Discussion}

All catecholamines studied were toxic in the same low concentration range of $10^{-5} \mathbf{M}$, and included NE, EPI, DA, isoproterenol, as well as 6-OHDA. Adrenergic agents that are not catecholamines, such as phenylephrine, clonidine, and terbutaline (data partially shown), were much less, if at all, toxic over the same concentration range. The fact that the beta-adrenergic agonist isoproterenol was toxic, and that the toxicity of NE was not blocked by the beta-adrenergic antagonist atenolol, argues against receptor mediation of NE toxicity. In the same culture system, the author has demonstrated a beta-adrenergic receptormediated phenomenon: NE at $10 \mu \mathrm{M}$ stimulates cAMP accumulation, and this effect is blocked by $33 \mu \mathrm{M}$ atenolol (data not shown). The similar toxicity between isoproterenol, which is not a substrate for MAO, and the endogenous catecholamines, which are, suggests that oxidative degradation by MAO does 


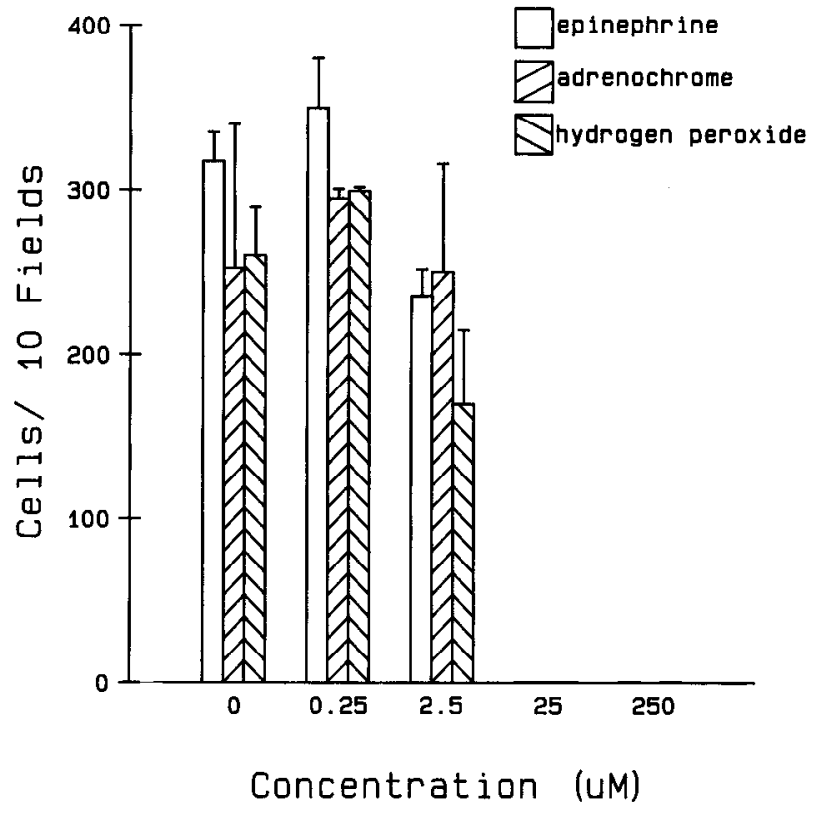

Figure 8. Effect of epinephrine and its auto-oxidation products on cerebral cortex in culture. Cultures were incubated with EPI and the products of EPI auto-oxidation, adrenochrome and hydrogen peroxide. EPI was made up and diluted in catecholamine vehicle. Adrenochrome (Sigma A 5877) was dissolved in 50\% ethanol to make a 10 mM solution. This was then diluted 10-fold with water, and subsequent dilutions were with $5 \%$ ethanol in order to keep the ethanol concentration approximately constant. A $30 \%$ solution of hydrogen peroxide was diluted with water to $100 \mathrm{~mm}$, Millipore filtered, and subsequently diluted to the appropriate concentrations with sterile water. Cultures were exposed to vehicles or the appropriate compounds in increasing concentrations as indicated. The experiment was set up in duplicate. No neurons survived in $25 \mu \mathrm{M}$ EPI, adrenochrome, or hydrogen peroxide.

not contribute significantly to catecholamine toxicity, at least in culture. On the other hand, auto-oxidation of catecholamines is likely to mediate the toxicity of these substances. This process is associated with the stoichiometric production of hydrogen peroxide, which is shown here to be toxic at the same concentration as catecholamines, and does not discriminate between isoproterenol and NE. The hypothesis that oxidative degradation of catecholamines is required for toxicity is further supported by the observation that catalase completely blocks the toxicity of NE. The sparing effect seen using catalase must be interpreted with caution, however, in view of the ability of catalase to function as a peroxidase (Aebi, 1974; for the oxidation of L-DOPA by HRP in the presence of hydrogen peroxide, see Waring, 1986). Hypothetically, catalase might act as a peroxidase, degrading $\mathrm{NE}$ and other catecholamines. However, studies of the effect of catalase on 6-OHDA auto-oxidation have shown that catalase, in fact, has no effect on the absolute amount of $p$-quinone formed, though does decrease its rate of formation (Sullivan et al., 1980). Studies by the author on the effect of catalase on the elimination of NE from growth medium showed similar results: in growth medium in the presence of catalase $(25 \mu \mathrm{cg} / \mathrm{ml}) 74 \%$ of NE (starting concentration $25 \mu \mathrm{M}$ ) remained at the end of $1 \mathrm{hr}$ as opposed to $54 \%$ remaining in the absence of catalase. It may be assumed therefore that the presence of catalase in the experiment reported in Figure 9 does not result in increased degradation of NE. In other experiments, catalase by itself was also shown to have no effect on cell survival in this culture system, in contrast to the results obtained by

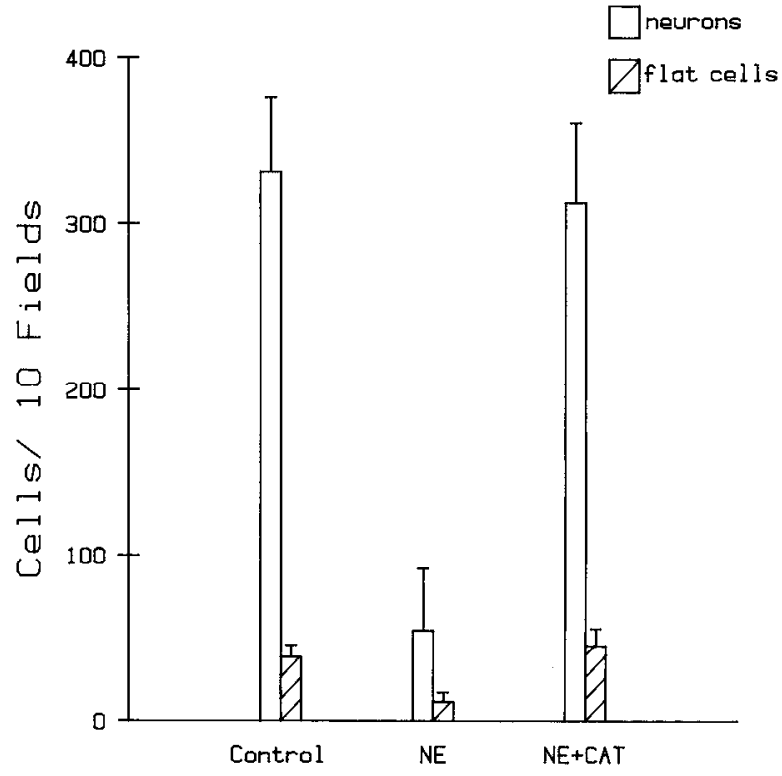

Figure 9. Protection of cerebral cortex from NE toxicity by catalase. Control cultures received only catecholamine vehicle $(12.5 \mu \mathrm{l} /$ well $)$ or water $(5 \mu \mathrm{l} /$ well). NE cultures received $12.5 \mu \mathrm{l}$ of $1 \mathrm{mM}$ NE for a final NE concentration of approximately $25 \mu \mathrm{M}$ plus $5 \mu \mathrm{l}$ water. $N E+C A T$ cultures received NE plus $5 \mu \mathrm{l}$ of a catalase solution, $1 \mathrm{mg} / \mathrm{ml}$, for a final catalase concentration of $10 \mu \mathrm{cg} / \mathrm{ml}$. In NE alone, $16 \%$ neurons survived. With the addition of catalase, however, $94 \%$ of ncurons present in the control cultures were present despite the addition of NE. This experiment was set up in quadruplicate.

Walicke et al. (1986) using cultures derived from embryonic chick forebrain and maintained in serum-free medium.

The toxicity of adrenochrome, however, may seem, at first, difficult to reconcile with a hypothesis that hydrogen peroxide is the toxic intermediate in NE toxicity. However, adrenochrome is unstable in aqueous solution, itself undergoing auto-oxidation (Graham, 1978). Therefore, it is not possible, on the basis of the current data, to assert that adrenochromc is ultimately the toxic species. It is also not possible to exclude the possibility that the toxicity of adrenochrome is itself mediated by the production of reactive oxygen intermediates. In fact, catalase was found to be able to at least partially block the toxicity of adrenochrome.

The toxicity of NE at the threshold concentration of $25 \mu \mathrm{M}$ required prolonged exposure (Fig. 4). The amount of NE remaining in growth medium was measured, starting with a concentration of $25 \mu \mathrm{M}$. It was found that, after $24 \mathrm{hr}, 2.2 \% \mathrm{NE}$ remained in medium incubated with cells and $0.4 \%$ remained in medium incubated without cells. The requirement for prolonged exposure to NE (and its degradation products) for the demonstration of toxicity, together with the rapid elimination of NE itself from the media, argues against toxicity's being an effect of NE and for toxicity's being an effect of one or more of the breakdown products. Toxicity at $250 \mu \mathrm{M}$ NE was manifest following an exposure of $1 \mathrm{hr}$ (Fig. 4). NE toxicity does develop rapidly, therefore, at higher concentrations.

NE toxicity is strongly dependent on culture density (Fig. 5), and is not demonstrable at $25 \mu \mathrm{M}$ NE unless the cultures are relatively sparse (11,000-28,000 cells/13 mm coverslip). At 250 $\mu \mathrm{M} \mathrm{NE}$, however, this effect of culture density is not seen, and no cells remain, even at platings at 900,000 cells/dish, yielding culture densities of approximately 68,000 cells/coverslip ( 2 ex- 


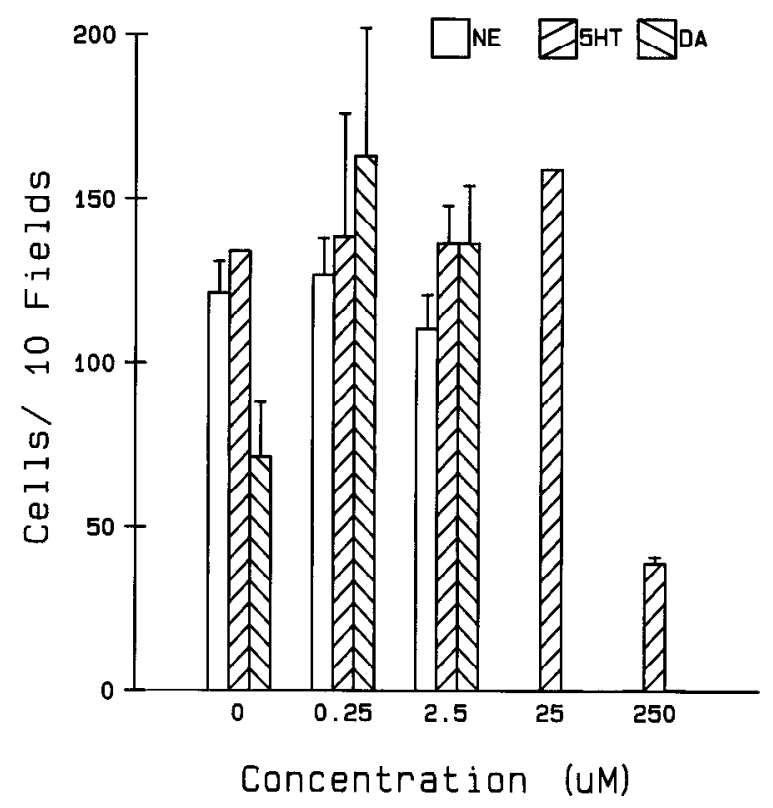

Figure 10. Effects of NE, DA, and serotonin on cerebral cortex in culture. The toxic effects of NE, DA, and serotonin were compared. All 3 monoamines were made up in catecholamine vehicle. The standard dose-response paradigm described for Figure I was used for each monoamine. In this experiment, no neurons survived in either $25 \mu \mathrm{M} \mathrm{NE}$ or DA. There was no reduction in the number of neurons surviving in serotonin at this concentration. At $250 \mu \mathrm{M}$ serotonin, surviving neurons were reduced to $30 \%$ of control cultures $(p<0.001)$.

periments). These results imply that protective mechanisms exist in cerebral cortex, at least in culture, to protect against catecholamine toxicity, and may be expected to include mechanisms for clearing and sequestering catecholamines, as well as for inactivating the toxic products of catecholamine degradation. These results also demonstrate that it is possible to overwhelm these protective mechanisms by increasing the catecholamine concentration. Unlike NE, 6-OHDA does not show this dependence of toxicity on culture density, and kills nearly all cells at $25 \mu \mathrm{M}$ even at the highest plating density tested $(900,000 \mathrm{cells} / \mathrm{dish}$; data not shown).

Is catecholamine toxicity plausible as a source of cell injury in the CNS? If so, then one might expect extracellular concentrations of catecholamines to attain those concentrations obscrved to produce toxicity in vitro. The concentration of NE in synaptic vesicles has been estimated at $10 \mathrm{~mm}$ (Beaudet and Descarries, 1984). The concentrations attained in the extracellular space depend on many factors, and, for the issue being considered here, tonic levels are probably more relevant than phasic levels. It may be reasonable to assume, in the absence of more direct evidence, that the concentration range in which NE produces a response in a target cell may be taken as a rough estimate of the concentration range encountered in vivo. NE has been shown to stimulate glycogenolysis in the cortical slice preparation with an $\mathrm{EC}_{50}$ of $0.5 \mu \mathrm{M}$ (Quach et al., 1978). Therefore we may expect that concentrations of NE of $0.5 \mu \mathrm{M}$ are encountered in vivo. In addition, the electrophysiological effects of NE that have been characterized in cerebral cortex have also been demonstrated in the low-micromolar concentration range (Madison and Nicoll, 1982). It is plausible that, under unusual circumstances, significantly highcr concentrations may be encountered: for example, during a seizure; during an acute psy- chotic episode; when uptake systems are blocked by the administration of tricyclic antidepressants; when degradation is blocked by MAO inhibitors; when drugs that cause release of catecholamines from presynaptic terminals, such as methylamphetamine, are abused; when catecholamine precursors, such as L-DOPA, are administered. However, given the dependence of catecholamine toxicity on culture density at $25 \mu \mathrm{M}$, and since in vivo conditions are notable for high cell density, one would expect that endogenous concentrations of catecholamines might have to get as high as $250 \mu \mathrm{M}$ to be toxic because, at this concentration, toxicity was observed even at the highest culture density tested. In comparison, the toxicity of glutamate occurs at concentrations of $10^{-4} \mathrm{M}$ or higher in cultures of mouse cerebral cortex (Choi, 1985; Rothman, 1985; and personal observations). This is approximately $4 \times$ the concentration of glutamate required to produce an electrophysiological response (personal observations).

These results have the further implication that 6-OHDA is not necessarily a specific toxin selective for catecholaminergic neurons (Malmfors and Thoenen, 1971; Trombley et al., 1986). The possibility of nonspecific effects of 6-OHDA is well known, since tissue damage is routinely observed surrounding injection sites in the CNS. This study demonstrates that nonspecific killing of both neurons and glia may occur at concentrations as low as $25 \mu \mathrm{M}$. Of particular note is the fact that, in the range of culture densities used in this study, a dependence of toxicity to 6-OHDA on culture density was not observed, in contrast to the toxicity of the endogenously occurring catecholamines. The possibility of toxic effects on noncatecholaminergic neurons, as well as glia, underlying some of the effects of 6-OHDA must be considered.

\section{References}

Aebi, H. (1974) Catalase. In Methods of Enzymatic Analysis, H. U. Bergmeyer, ed., pp. 673-684, Academic, New York.

Beal, M. F., N. W. Kowall, D. W. Ellison, M. F. Mazurek, K. J. Swartz, and J. B. Martin (1986) Replication of the neurochemical characteristics of Huntington's disease by quinolinic acid. Nature $321: 168-$ 171.

Beaudet, A., and L. Descarries (1984) Fine structure of monoamine axon terminals in cerebral cortex. In Monoamine Innervation of $\mathrm{Ce}$ rebral Cortex, L. Descarries, T. R. Reader, and H. H. Jasper, eds., pp. 109-127, Liss, New York.

Bignami, A., T. Raju, and D. Dahl (1982) Localization of vimentin, the nonspecific intermediate filament protein, in embryonal glia and in early differentiating neurons: In vivo and in vitro immunofluorescence study of the rat embryo with vimentin and neurofilament antisera. Dev. Biol. 91: 286-295.

Choi, D. W. (1985) Glutamate neurotoxicity in cortical cell culture in calcium-dependent. Neurosci. Lett. 58: 293-297.

Dahl, D., D. C. Rueger, A. Bignami, K. Weber, and M. Osborn (1981) Vimentin, the 57,000 molecular weight protein of fibroblast filaments, is the major cytoskeletal component of immature glia. Eur. J. Cell Biol. 24: 191-196.

D'Amato, R. J. D., Z. P. Lipman, and S. H. Snyder (1986) Selectivity of the Parkinsonian neurotoxin MPTP: Toxic metabolite MPP + binds to neuromelanin. Science 231: 987-989.

Dichter, M. A. (1978) Rat cortical neurons in cell culture: Culture methods, cell morphology, electrophysiology, and synapse formation. Brain Res. 149: 279-293.

Ferrante, R. J., N. W. Kowall, M. F. Beal, E. P. Richardson, Jr., E. D. Bird, and J. B. Martin (1985) Selective sparing of a class of striatal neurons in Huntington's disease. Science 230: 561-563.

Franke, W. W., E. Schmid, M. Osborn, and K. Weber (1978) Different intermediate-sized filaments distinguished by immunofluorescence microscopy. Proc. Natl. Acad. Sci. USA 75: 5034-5038.

Graham, D. G. (1978) Oxidative pathways for catecholamines in the 
genesis of neuromelanin and cytotoxic quinones. Mol. Pharmacol. 14: 633-643.

Graham, D. G. (1984) Catecholamine toxicity: A proposal for the molecular pathogenesis of manganese neurotoxicity and Parkinson's disease. Neurotoxicology 5: 83-95.

Heacock, R. A. (1959) The chemistry of adrenochrome and related compounds. Chem. Rev. 59: 181-237.

Heikkila, R. E., and G. Cohen (1973) 6-Hydroxydopamine: Evidence for superoxide radical as an oxidative intermediate. Science $181: 456-$ 457.

Javitch, J. J., R. J. D'Amato, S. M. Strittmatter, and S. H. Snyder (1985) Parkinsonism-inducing neurotoxin, $N$-methyl-4-phenyl-1,2,3,6-tetrahydropyridine: Uptake of the metabolite $N$-methyl-4-phenylpyridine by dopamine neurons explains selective toxicity. Proc. Natl. Acad. Sci. USA 82: 2173-2177.

Langston, J. W., P. Ballard, J. W. Tetrud, and I. Irwin (1983) Chronic Parkinsonism in humans due to a product of meperidine-analog synthesis. Science 219: 979-980.

Liang, Y.-O., R. M. Wightman, and R. N. Adams (1976) Competitive oxidation of 6-hydroxydopamine by oxygen and hydrogen peroxide. Eur. J. Pharmacol. 36: 455-458.

Madison, D. V., and R. A. Nicoll (1982) Noradrenaline blocks accommodation of pyramidal cell discharge in the hippocampus. Nature 299: 636-638.

Malmfors, T., and H. Thoenen (eds.) (1971) 6-Hydroxydopamine and Catecholamine Neurons, North-Holland, Amsterdam.

Marker, H. S., C. Weiss, D. J. Silides, and G. Cohen (1981) Coupling of dopamine oxidation (monoamine oxidase activity) to glutathione oxidation via the generation of hydrogen peroxide in rat brain homogenates. J. Neurochem 36: 589-593.

Olney, J. W. (1978) Neurotoxicity of excitatory amino acids. In Kainic Acid as a Tool in Neurobiology, E. G. McGeer, J. W. Olney, and P. L. McGeer, eds., pp. 95-121, Raven, New York.

Pixley, S. K., and C. W. Cotman (1985) Laminin supports short-term survival of rat septal neurons in low-density, serum-free cultures. J. Neurosci. Res. 15: 1-17.

Quach, T. T., C. Rose, and J.-C. Schwartz (1978) [ $\left.{ }^{3} \mathrm{H}\right]$ Glycogen hydrolysis in brain slices: Responses to neurotransmitters and modulation of noradrenaline receptors. J. Neurochem. 30: 1335-1341.

Raff, M. C., K. L. Fields, S.-I. Hakomori, R. Mirsky, R. M. Pruss, and J. Winter (1979) Cell-type specific markers for distinguishing and studying neurons and the major classes of glial cells in culture. Brain Res. 174: 283-308.
Rothman, S. M. (1985) The neurotoxicity of excitatory amino acids is produced by passive chloride flux. J. Neurosci. 5: 1483-1489.

Saner, A., and H. Thoenen (1970) Model experiments on the molecular mechanism of action of 6-hydroxydopamine. Mol. Pharmacol. 7: 147-154.

Senoh, S., and B. Witkop (1959) Non-enzymatic conversions of dopamine to norepinephrine and trihydroxyphenethylamines. J. Am. Chem. Soc. 81: 6222-6235.

Senoh, S., C. R. Creveling, S. Udenfreund, and B. Witkop (1959) Chemical, enzymatic, and metabolic studies on the mechanism of oxidation of dopamine. J. Am. Chem. Soc. 81: 6236-6240.

Snodgrass, S. R., W. F. White, B. Biales, and M. Dichter (1980) Biochemical correlates of GABA function in rat cortical neurons in culture. Brain Res. 190: 123-138.

Sullivan, S. G., L. Sudarsky, and A. Stern (1980) Potentiating effect of catalase on inhibition by superoxide dismutase of 6-hydroxydopamine autoxidation. In Chemical and Biochemical Aspects of Superoxide Dismutase, J. V. Bannister and H. A. O. Hill, eds., pp. 364371, Elsevier/North Holland, New York.

Suter, W., and I. Matter-Jaeger (1984) Genotoxicity of apomorphine and various catecholamines in the Salmonella mutagenicity test (Ames test) and in tests for primary DNA damage using DNA repair-deficient $B$. subtilis strains (Rec assay). Mutat. Res. 137: 17-28.

Trombley, E., E. Allen, J. Soyke, C. D. Blaha, K. F. Lane, and B. Gordon (1986) Doses of 6-hydroxydopamine sufficient to deplete norepinephrine are not sufficient to decrease plasticity in the visual cortex. J. Neurosci. 6: 266-273.

Ungerstedt, U. (1971) Use of intracerebral injection of 6-hydroxydopamine as a tool for morphological and functional studies on central catecholamine neurons. In 6-Hydroxydopamine and Catecholamine Neurons, T. Malmfors and H. Thoenen, eds., pp. 315-332, NorthHolland, Amsterdam.

Walicke, P., S. Varon, and M. Manthorpe (1986) Purification of a human red blood cell protein supporting the survival of cultured CNS neurons, and its identification as catalase. J. Neurosci. 6: 1114-1121.

Waring, P. (1986) The time-dependent inactivation of human brain dihydropteridine reductase by the oxidation products of L-DOPA. Eur. J. Biochem. 155: 305-310.

Wheatley, A. M., F. T. Thandroyen, and L. H. Opie (1985) Catecholamine-induced myocardial cell damage: Catecholamines or adrenochrome. J. Mol. Cell. Cardiol. 17: 349-359. 